\title{
RECOVERY OF MYELINATION IN RAT OPTIC NERVE AFTER DEVELOPMENTAL RETARDATION BY CORTISOL ${ }^{1}$
}

\author{
MARTHA C. BOHN² AND VICTOR L. FRIEDRICH, JR. \\ Laboratory of Neuromorphology, Department of Biobehavioral Sciences, University of Connecticut, Storrs, Connecticut 06268
}

Received October 19, 1981; Revised March 15, 1982; Accepted April 20, 1982

\begin{abstract}
Oligodendrocyte formation and myelination in the optic nerve of rats treated with cortisol on postnatal days 7 to 18 were investigated at 21 and 60 days by autoradiographic and morphometric methods.

At 21 days, the amount of myelin in the optic nerve of treated rats was reduced by $42 \%$. This reduction was accompanied by a $39 \%$ decrease in the number of myelinated axons. In addition, the myelin/axon area ratio was reduced $22 \%$ for those axons which were myelinated.

At 60 days, the amount of myelin, the number of axons myelinated, the average axonal size, and the average amount of myelin per axon had completely recovered to normal values. Tritiated thymidine-labeling experiments showed that the genesis of oligodendrocytes was enhanced following the cessation of treatment. Since the number of oligodendrocytes in the optic nerve of treated rats was normal at 60 days, it is suggested that the retarding effect of cortisol on myelination in the optic nerve may be mediated, at least in part, by inhibited formation of oligodendrocytes during treatment.
\end{abstract}

Treatment of immature rats with corticosteroids inhibits cell proliferation in postnatal germinal zones in the brain, resulting in permanent numerical deficits of cerebellar microneurons as well as reduced cerebral weight and DNA content (Cotterrell et al., 1972; Howard, 1973; Clos et al., 1975; Bohn and Lauder, 1978, 1980). While it is clear that postnatal acquisition of neurons is affected by corticosteroids, equivalent effects on gliogenesis have been suggested (Howard and Benjamins, 1975; Gumbinas et al., 1973) but not demonstrated.

Hormonal interference with the processes of gliogenesis and glial cell differentiation, including myelin production, could contribute to some of the behavioral changes resulting from neonatal glucocorticoid treatment. For example, it has been observed that cortisol treatment at birth delays the development of cortical evoked potentials in response to sensory stimuli (Salas and Schapiro, 1970), changes the development of the startle reflex seizure patterns (Schapiro, 1968; Vernadakis and Woodbury, 1963), and produces adult animals which are emotionally labile and hyper-responsive (Olton et al., 1974).

\footnotetext{
${ }^{1}$ This work was supported by Public Health Service Grants NS09904 and MH-05572. We wish to thank Dr. Enrico Mugnaini for the excellent facilities used in this work. The technical assistance of Mrs. Mary Goss is also gratefully acknowledged.

${ }^{2}$ To whom correspondence should be addressed at her present address: Department of Neurology, Cornell Medical College, 515 East 71st Street, New York, NY 10021.
}

Glucocorticoids also have been observed to regulate enzymes localized in glia, including glycerol phosphate dehydrogenase (DeVellis and Inglish, 1973), glutamine synthetase (Juulink et al., 1981), 2',3'-cyclic nucleotide $3^{\prime}$-phosphohydrolase (Waziri and Sahu, 1980), and 3'phosphoadenosine 5 -phosphosulfate:galactosylceramide sulfotransferase (Dawson and Kernes, 1979).

While these diverse observations suggest that glucocorticoids may influence the differentiation of glial cells, little morphological information is available to support this possibility. The present study was undertaken to obtain quantitative measurements on the in vivo effects of cortisol on myelination and gliogenesis. The optic nerve was chosen for study because the components of the optic nerve are simple in comparison to other central myelinated tracts. Furthermore, gliogenesis and the initiation of myelination take place postnatally and have been studied carefully in the optic nerve of the normal rat (Skoff et al., 1976a, b, 1980; Tennekoon et al., 1977, 1980; Vaughn and Peters, 1967; Vaughn, 1969). Here, we have compared the optic nerves from normal and cortisol-treated rats to assess the acute and chronic effects on the generation of oligodendrocytes, the total amount of myelin, and the amount of myelin per retinal ganglion cell axon.

\section{Materials and Methods}

Preparation of specimens. Pregnant Long-Evans rats (Blue Spruce Farms, Altamont, NY) were obtained at 14 
days of gestation and maintained on a 13:11-hr light:dark cycle with food and water ad libitum. Pups were pooled on day 1 after birth and divided into seven litters consisting of 8 pups with comparable total body weight. Only male pups were used for data analysis. Three litters were injected once daily from postnatal days 7 to 18 with cortisol suspended in $1 \%$ carboxymethyl cellulose at a dose of $10 \mu \mathrm{g} / \mathrm{gm}$ of body weight (limited to a maximum dose of $200 \mu \mathrm{g} /$ day). Four litters remained untreated and were used as controls. We chose to use normal, rather than vehicle-injected, pups as controls to avoid the possible effects of increased endogenous levels of glucocorticoids caused by the stress of repeated injections. Some pups were sacrificed at 21 days after birth; the remainder were weaned at 30 days and sacrificed on postnatal day 60 . At 60 days, the body weight of treated animals was normal although the hrain weight remained depressed by $15 \%$ as reported previously (Bohn and Lauder, 1980).

All animals were sacrificed under chloral hydrate anesthesia by vascular perfusion with mixtures containing $4 \%$ formaldehyde and $0.5 \%$ glutaraldehyde (21-day rats) or $1 \%$ formaldehyde and $2 \%$ glutaraldehyde (60-day rats) in $0.12 \mathrm{M}$ phosphate buffer, $\mathrm{pH} 7.3$, stored overnight at $4^{\circ} \mathrm{C}$ and dissected the following day. Tissue pieces were postfixed in phosphate-buffered osmium tetroxide followed by uranyl acetate and embedded in a TAAB Epon embedding mixture.

Morphometry. One optic nerve from each animal was sectioned transversely for light and electron microscopy at a level midway between the optic chiasm and the optic foramen. Semithin sections for area measurements contained the entire cross-section of each nerve as did thin sections of the 21-day material. Thin sections of the 60day material contained at least half of the cross-section of the nerve in each case.

The total cross-sectional area of each nerve was determined by planimetry of drawings of $2-\mu \mathrm{m}$-thick sections stained with toluidine blue. The drawings were made with a camera lucida and calibrated with a stage micrometer.

Silver transverse sections were mounted for electron microscopy on 300 mesh grids and stained with uranyl acetate and lead citrate. One electron micrograph was taken at $\times 4,000$ in each of every grid square from a single section from each animal and printed at $\times 10,000$ final magnification for analysis. The positions within the grid squares of the fields photographed were arbitrary, except that fields containing substantial $(>30 \%)$ areas of glial perikarya were rejected. Each animal was represented by 5 to 10 micrographs, covering an area of 680 to $1,360 \mu \mathrm{m}^{2}$ and representing 0.2 to $0.6 \%$ of the transverse area of the optic nerve at the level studied.

The fractional areas of unmyelinated $\left(A_{A}^{\text {uax }}\right)$ and myelinated $\left(A_{A}^{\max }\right)$ axons (comprised of axons but not the surrounding myelin sheaths) and of myelin $\left(A_{A}^{\mathrm{my}}\right.$; comprised of compact myelin but neither the included axoplasm nor the associated oligodendrocyte cytoplasm) were determined by point hit counting (as described by Williams, 1977), using a regular array of 100 points superimposed over each micrograph.

In addition, the number of axons per unit area was determined by counting the axons in each micrograph.
This parameter was determined separately for unmyelinated $\left(N_{A}^{\text {uax }}\right)$ and myelinated $\left(N_{A}^{\max }\right)$ axons. The average cross-sectional areas of unmyelinated $\left(\bar{a}^{\text {uax }}\right)$ and myelinated $\left(\bar{a}^{\mathrm{max}}\right)$ axons were estimated by

$$
\bar{a}^{\text {uax }}=\frac{A_{A}^{\text {uax }}}{N_{A}^{\text {uax }}} \text { and } \bar{a}^{\max }=\frac{A_{A}^{\max }}{N_{A}^{\max }}
$$
by

The area of myelin per myelinated axon was estimated

$$
A_{\max }^{\operatorname{my}}=\frac{A_{A}^{\operatorname{my}}}{N_{A}^{\max }}
$$

and the total area of myelin $\left(A^{\mathrm{my}}\right)$ was estimated by

$$
A^{\mathrm{my}}=A_{A}^{\mathrm{my}} A^{T}
$$

where $A^{T}$ is the total area of the nerve in cross-section.

Autoradiography. Included in the above analysis were 4 cortisol-treated and 2 normal rats which were given a single injection of $\left[{ }^{3} \mathrm{H}\right]$ thymidine, $10 \mu \mathrm{Ci} / \mathrm{gm}$ of body weight, at 19 days after birth and were sacrificed at 60 days after birth as described above. Autoradiographic analysis of these 6 rats was performed on $2-\mu \mathrm{m}$-thick, longitudinal sections of the intracranial part of the optic nerve from these animals. These longitudinal sections were obtained from the nerve contralateral to that sectioned transversely for the above morphometric analysis. Sections were mounted on glass slides, coated with emulsion, and exposed at $4^{\circ} \mathrm{C}$ for 3 months. Slides were developed with D-19, fixed with Kodak fixer, stained with toluidine blue, and coverslipped with a TAAB Epon embedding mixture.

The numbers of unlabeled ( 0 to 4 grains), lightly labeled ( 5 to 50 grains), and heavily labeled (more than 50 grains) glial cell nuclei were determined by counting at $\times 1,000$ under oil and tabulated separately for oligodendrocytes and astrocytes. Labeling indices were determined by expressing the number of labeled cells of each type (astrocytes or oligodendrocytes) as percentages of the total number of cells of that type. Astrocytes and oligodendrocytes were readily distinguished (Skoff et al., 1976b) and were counted separately. Microglial and unclassified nuclei were excluded; they accounted for less than $3 \%$ of all nuclei. Two hundred to 250 nuclei from each specimen were examined and data were collected in a blind experimental fashion by having the slides coded.

Statistics. Data were reduced to a single estimate of each parameter studied for each rat. The counting fractions $\left(A_{A}\right.$ and $\left.N_{A}\right)$ were estimated for each animal by summing the counts from all micrographs before performing the appropriate divisions.

The values of the six parameters directly determinedthe total nerve cross-sectional area, the area fractions of myelin, myelinated, and unmyelinated axons, and the numbers of myelinated and unmyelinated axons per unit area-were analyzed by multivariate analysis of variance using the procedure GLM of the statistical package from SAS (Helwig and Council, 1979). The decision was crossed in age and treatment with interaction; the analysis was restricted to six variables because of the small number of observations (13 rats total). The results showed a significant effect of treatment $(p=0.015)$ and a significant interaction of treatment with age $(p=0.017$; 
Wilks criterion; Helwig and Council, 1979). This indicates that the optic nerves of the cortisol-treated rats differed from normal in at least one of the parameters studied and that the amount of the difference changes with age.

Paired comparisons between values from treated and normal animals were performed for selected parameters by $t$ tests using the SAS procedure TTEST (Helwig and Council, 1979). This procedure tests for equality of variance for each comparison; equality of variance was accepted for pairs with $p>0.10$ on the variance test, and the unequal variance test was used for pairs failing to meet this criterion. Differences were accepted as significant (Tables I and II) at a comparison-wise error rate of 0.05 ; no correction was made for the multiplicity of comparisons.

\section{Results}

Myelination at 21 days. Optic nerves were examined at 21 days to assess the immediate effects of cortisol treatment on myelination. Electron micrographs showed a clearly reduced content of myelin in cortisol-treated as compared to normal rats (Fig. 1). The quantitative analysis (Table I) revealed that the total cross-sectional area of the nerve was reduced by $24 \%$, the area fraction of myelin was decreased by $25 \%$, and the total cross-sectional area of myelin was lowered by $42 \%$. The drastic reduction in total myelin area was accompanied by an equal reduction (39\%) in the proportion of axons myelinated, from $74 \%$ in the normal animals to $46 \%$ in the treated animals. The area of myelin per myelinated axon was normal.

Unmyelinated axons were of equal size in the two groups; however, the myelinated axons were $21 \%$ larger in the treated as compared to normal animals (Table I). The ratio, area of myelin to area of myelinated axons, was reduced significantly in the treated animals, suggesting that the myelin sheaths in the treated animals were thinner than normal for the same size axon.
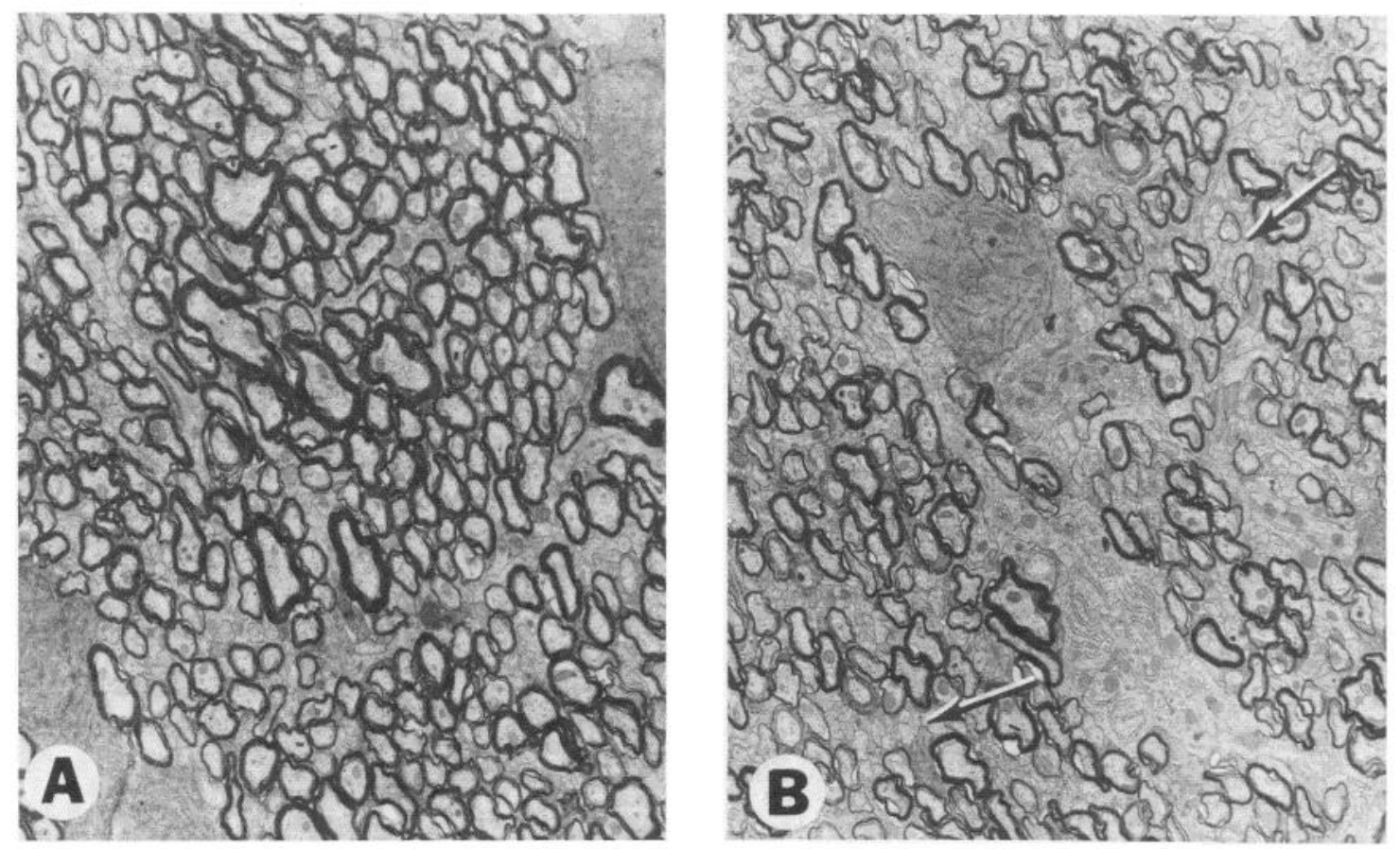

Figure 1. Cross-section of the optic nerve at 21 days of age from $(A)$ a normal rat and $(B)$ a rat treated with cortisol on postnatal days 7 to 18 . Whereas most axons in the normal nerve are surrounded by compact myelin, many groups of axons remain unmyelinated (arrows) in the treated nerve. Magnification $\times 4,800$.

TABLE I

Optic nerve in cross-section at 21 days after birth

The values are the mean \pm SEM from 3 animals for each treatment from counts of 700 to 1,000 grid intersections and 900 to 1,400 axon profiles from each animal. NSD indicates no significant difference $(p>0.05 ; t$ test); for all other differences, $p \leq 0.05$. The myelin/axon ratio is the area fraction of myelin divided by the area fraction of myelinated axons.

\begin{tabular}{|c|c|c|c|}
\hline & Normal & Cortisol & Effect \\
\hline & & & $\%$ \\
\hline Myelin area fraction $(\%)$ & $26 \pm 2$ & $20 \pm 1$ & -25 \\
\hline Percentage of axons myelinated & $74 \pm 3$ & $46 \pm 1$ & -39 \\
\hline Area of myelin per myelinated axon $\left(\mu \mathrm{m}^{2}\right)$ & $0.26 \pm 0.01$ & $0.24 \pm 0.01$ & NSD \\
\hline Average size of myelinated axons $\left(\mu \mathrm{m}^{2}\right)$ & $0.40 \pm 0.01$ & $0.48 \pm 0.03$ & 21 \\
\hline
\end{tabular}



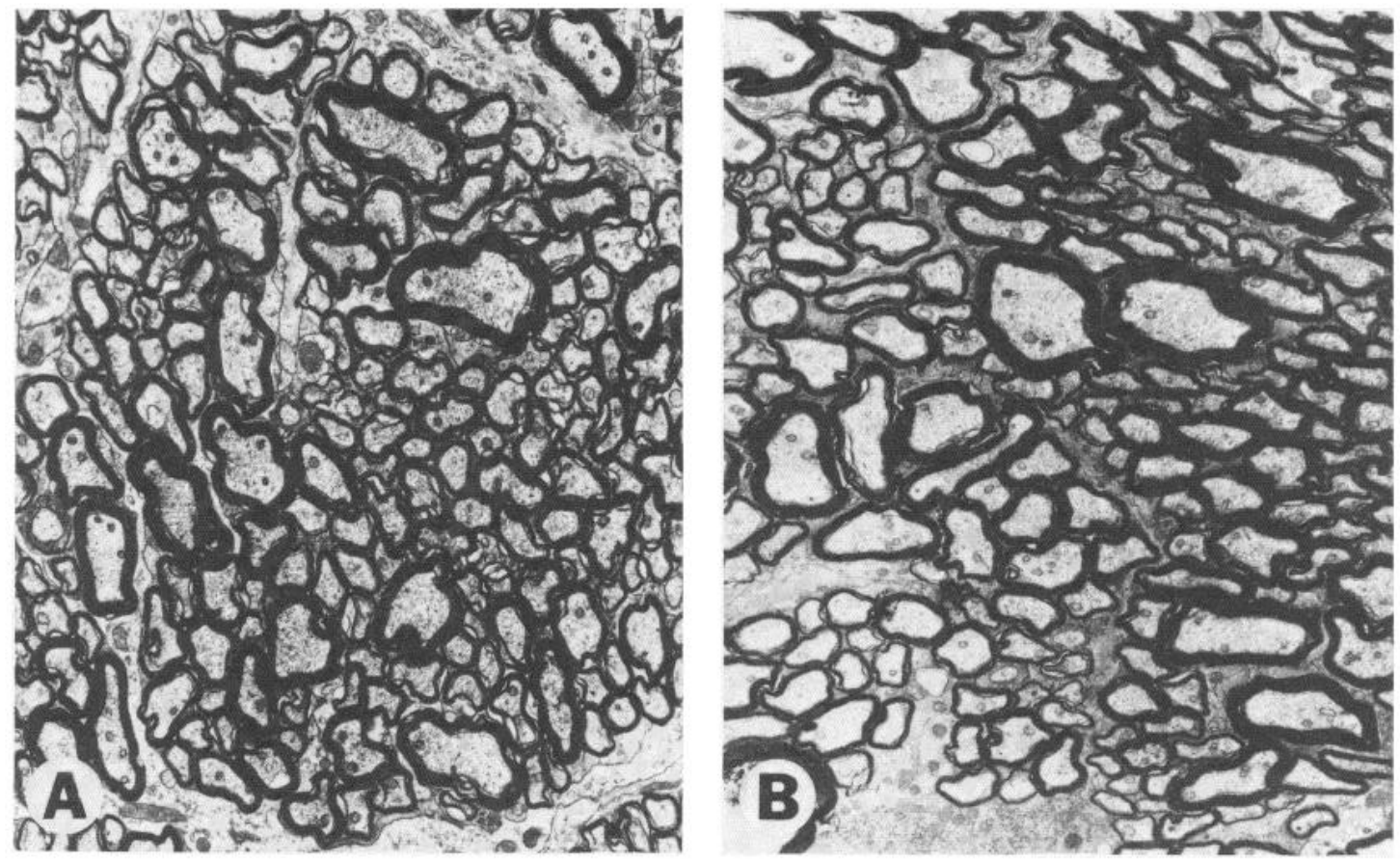

Figure 2. Cross-section of optic nerve at 60 days of age from $(A)$ a normal rat and $(B)$ a rat treated with cortisol on postnatal days 7 to 18 . Magnification $\times 4,800$.

TABLE II

Optic nerve in cross-section at 60 days after birth

The values are the mean \pm SEM from 4 normal and 3 cortisol-treated animals from counts of 500 to 1,000 grid intersections and 800 to 1,400 axon profiles from each animal. NSD indicates no significant difference ( $p>0.05 ; t$ test). The myelin/axon area ratio is the area fraction of myelin divided by the area fraction of myelinated axons.

\begin{tabular}{lccc}
\hline & Normal & Cortisol & Effect \\
\hline Total area $\left(\mathrm{mm}^{2}\right)$ & $0.316 \pm 0.006$ & $0.305 \pm 0.012$ & NSD \\
Myelin area fraction $(\%)$ & $31.8 \pm 0.8$ & $30.4 \pm 1.4$ & NSD \\
Total myelin area $\left(\mathrm{mm}^{2}\right)$ & $0.100 \pm 0.003$ & $0.093 \pm 0.005$ & NSD \\
Percentage of axons myelinated & 100 & 100 & NSD \\
Area of myelin per myelinated axon $\left(\mu \mathrm{m}^{2}\right)$ & $0.51 \pm 0.07$ & $0.47 \pm 0.03$ & NSD \\
Average size of myelinated axons $\left(\mu \mathrm{m}^{2}\right)$ & $0.74 \pm 0.08$ & $0.66 \pm 0.05$ & NSD \\
Myelin/axon area ratio & $0.69 \pm 0.04$ & $0.71 \pm 0.04$ & NSD \\
\hline
\end{tabular}

Myelination at 60 days. To determine whether the inhibition of myelination observed at 21 days was a permanent or reversible effect, we looked at the optic nerves of adult treated and normal rats. At 60 days, the optic nerves of the cortisol-treated animals appeared normal in all respects (Fig. 2). No significant differences in the total cross-sectional area of the nerve, the total cross-sectional area of myelin, or the myelin area fraction were observed (Table II). In contrast to the deficit of myelinated axons at 21 days, the proportion of axons myelinated was the same (100\%) in both cortisol-treated and normal animals at 60 days after birth as were the average amount of myelin per myelinated axon, the average size of the axons, and the ratio, area of myelin to area of myelinated axon. These results demonstrate a substantial recovery of myelination after the cessation of cortisol treatment at 18 days after birth.

Glial cell numbers and thymidine labeling. Genesis of most oligodendrocytes in the optic nerve occurs during the 2nd and 3rd postnatal weeks (Skoff et al., 1976b); our schedule of cortisol treatment, from day 7 to 18 , coincided with this period. In our material, injection of $\left[{ }^{3} \mathrm{H}\right]$ thy- midine on day 19 resulted in the labeling of $12 \pm 2 \%$ (mean \pm SEM) of the oligodendrocytes in normal rats at 60 days and in the labeling of twice as many $(22 \pm 2 \% ; p$ $<0.01 ; t$ test) of the oligodendrocytes in the cortisoltreated rats, suggesting that the period during which oligodendrocytes were formed was effectively prolonged by cortisol treatment (or cessation of treatment). In the same specimens, $3 \%$ of the astrocytes were labeled in the treated animals and no astrocytes were labeled in the normal animals.

In the 60-day-old animals, the number of oligodendrocytes per unit area of nerve was similar in treated and normal animals $\left(691 \pm 43 / \mathrm{mm}^{2}\right.$, treated; $794 \pm 46 / \mathrm{mm}^{2}$, normal; mean \pm SEM; $p=0.12$ ). Since the total crosssectional area of the nerve was also normal in the 60-dayold treated animals (Table II), it appears that the total number of oligodendrocytes was not abnormal at 60 days.

\section{Discussion}

Developmental studies of the rat optic nerve have demonstrated that the three main cell types comprising the nerve are formed over different intervals. The retinal 
ganglion cells are formed entirely during the prenatal period (Sidman, 1961). Astrocytes are formed postnatally, with their genesis nearly complete by day 10 , while oligodendrocytes, the cells which myelinate the ganglion cell axons, are formed primarily during the 2nd and 3rd postnatal weeks (Skoff et al., 1976a, b). Myelin sheath formation also occurs at maximal rates during the 2 nd and 3rd postnatal weeks and subsides thereafter (Vaughn, 1969; Hirose and Bass, 1973; Skoff et al., 1976b, 1980; Tennekoon et al., 1977). In the present study, we treated rats with cortisol from postnatal days 7 to 18 and examined the animals at postnatal day 21 to assess the acute effects of the treatment and at day 60 to assess the long term effects. This treatment was chosen specifically to coincide with the period during which oligodendrocyte precursor cells were dividing and myelin formation was intense.

The cortisol treatment caused decreases of $25 \%$ in the myelin area fraction, $42 \%$ in the total area fraction of myelin, and $39 \%$ in the proportion of axons myelinated as observed shortly after the cessation of treatment (21day-old animals). No change occurred in the total number of axons in the nerve (M. C. Bohn and V. L. Friedrich, Jr., unpublished data). We found no myelin debris or other evidence for degeneration of myelin sheaths in the 21-day-old animals; it thus appears that the effect of the cortisol treatment was to block the formation of myelin sheaths rather than to cause their degeneration.

When examined 6 weeks after the cessation of treatment, the treated animals showed normal myelination. This full recovery in myelination was accompanied by enhanced formation of oligodendrocytes after the cessation of treatment.

Increased proliferation of oligodendrocytes after cessation of treatment. We found that $12 \%$ of the oligodendrocytes were labeled at postnatal day 60 after a single injection of $\left[{ }^{3} \mathrm{H}\right]$ thymidine at day 19 . This number includes both heavily and lightly labeled cells and thus comprises most cells in the $S$ phase at the time of injection and their progeny. In populations which divide many times after thymidine administration, dilution of the label through mitosis may reduce the number of labeled cells visualized. In the present case, thymidine was injected well after the peak age of oligodendrogliogenesis (10 days; Skoff et al., 1976b) and long exposure times were employed to enhance autoradiographic sensitivity. For these reasons, we believe that the dilution effect is negligible for our data from normal animals.

Skoff et al. (1976b) reported a total of about 8 labeled oligodendrocytes per cross-section of optic nerve when rats were injected with thymidine at postnatal day 17 and sacrificed at day 30 . Our figure of $12 \%$ of the oligodendrocytes labeled after thymidine injection at day 19 and sacrificed at day 60 corresponds to about 22 labeled cells per cross-section. The greater number of cells labeled in our study probably reflects our longer interval between injection and sacrifice (41 days as compared to 13 days), which allowed more divisions of labeled precursor cells. In addition, we employed longer exposure times for our autoradiograms, an approach which may increase the yield of labeled cells severalfold (Skoff et al., 1976b).

Generation of oligodendrocytes in the rat optic nerve continues at least through postnatal days 30 to 35 (Skoff et al., 1976b). Our single injection should have labeled only a fraction of the cells proliferating at day 19 , those in the $S$ phase of the cell cycle at the time of injection. Thus, our results indicate that a substantial proportion of oligodendrocytes, perhaps $25 \%$ or more, are formed well after the age at which oligodendrogliogenesis is maximal.

In cortisol-treated rats, $22 \%$ of the oligodendrocytes were labeled at day 60 after a single injection of $\left[{ }^{3} \mathrm{H}\right]$ thymidine at day 19 , nearly twice the normal value. This result indicates either that an excess of cells were in the $S$ phase at day 19 , that those cells which were in the $S$ phase at that time divided excessively between days 21 and 60 , or both. If excessive, repeated passages through the mitotic cycle did occur among cells of the cortisoltreated animals, then the dilution effect may cause our data to underestimate the extent of oligodendrocyte proliferation in the treated animals. Thus, while the exact amount remains to be established, our results clearly demonstrate that a stimulation of oligodendrogliogenesis occurs after the cessation of treatment. Its minimum value is about an $80 \%$ increase over the normal rate.

Mechanisms of action. Myelination is a complex event involving both axons and neuroglial cells. It is presently unclear whether the inhibition of myelin formation by cortisol treatment results from action on either or both of these potential cellular targets.

The reduction in myelination that we observed at 21 days may have resulted from the formation of fewer oligodendrocytes during the period of cortisol administration or from an impairment of their capacity to differentiate and form myelin. These might be direct effects on glial cells, since glucocorticoid receptors have been reported in glial cells in the optic nerve (Meyer and McEwen, 1982). Our concomitant findings of enhanced proliferation of oligodendrocytes between days 21 and 60 and of normal cell numbers at day 60 suggest that the number of oligodendrocytes was reduced during cortisol treatment. Such a reduction might occur through enhanced cell death or by inhibition of cell proliferation.

The proliferation of glial cell precursors appears to be inhibited by high levels of glucocorticoids both in vivo and in vitro (Howard, 1973; Howard and Benjamins, 1975; Cotterrell et al., 1972; Grasso and Johnson, 1977). Cell proliferation in the subependymal layer, an important source of postnatally formed glial cells (Smart, 1961; Privat and Leblond, 1972) is inhibited during cortisol treatment and rebounds above control levels following termination of the treatment (Bohn, 1979). The occurrence of a similar rebound phenomenon in the optic nerve could account for our observation of enhancement of oligodendrocyte formation after the cessation of cortisol treatment.

A second possible mechanism for the inhibition of myelination is suggested by the present results. At 21 days after birth, nearly twice as many axons are unmyelinated in the treated as compared to the control animals (Table I). Myelinated axons are substantially larger than unmyelinated ones in both normal and treated animals (Table I); this implies that cortisol treatment results in an abnormally high proportion of small axons in the optic 
nerve. This result, which must be considered preliminary pending confirmation by direct analysis of the sizes of individual axons, suggests an inhibition of axonal enlargement by the hormone, perhaps reflecting a general inhibition of protein synthesis. This effect in itself could account for the inhibition of myelination, since axonal size or some other property correlated with size may be critical in stimulating ensheathment (Matthew and Duncan, 1971). However, results from transplantation studies suggest that glial cells might influence axonal size (Aguayo et al., 1979); it thus remains possible that the excess of small axons in our treated animals is secondary to impaired neuroglial function rather than its cause.

Recovery of myelination. Gumbinas et al. (1973) have analyzed the effect of corticosteroid treatment on the development of the pyramidal tract. A single injection of prednisone to 6 -day-old rats caused a $44 \%$ reduction in the fraction of axons myelinated at 21 days after birth, a result similar to ours in optic nerve after more prolonged treatment with cortisol. At 42 days after birth, they found some improvement, but the treated animals were still significantly less myelinated than normal. By contrast, we found virtually complete recovery in our older animals. This difference may reflect differences in the particular steroid injected, in the schedule of treatment, or in the ages at which the animals were examined. In addition, it is possible that fiber tracts differ in their capacity to recover from early corticosteroid treatment.

In this study, the cessation of cortisol treatment was followed not only by recovery of myelination but also by extended genesis of oligodendrocytes as demonstrated by thymidine autoradiography. A similar association of oligodendrogliogenesis with recovery of myelination has been demonstrated after demyelination induced by virus inoculation (Herndon et al., 1977) and by treatment with Cuprizone (Ludwin, 1979). These experiments involved the initial destruction of oligodendrocytes and thereby created a deficit of oligodendrocytes. It remains to be established whether the cortisol treatment caused a deficit at 21 days in the number of oligodendrocytes.

An analysis of remyelination after Cuprizone treatment has led to the suggestion that a signal for the generation of oligodendrocytes may result from local tissue reaction to demyelination (Ludwin, 1980). In the present case, we have found that stimulation of excessive oligodendrocyte proliferation can occur in the absence of substantial myelin degeneration. While our results do not exclude a role for degeneration in stimulating oligodendrogliogenesis, they do suggest that, at least in young animals, myelin degeneration is not necessary for such stimulation to occur.

The effects that we observed following neonatal cortisol treatment could be mediated indirectly through changes in other hormonal systems. Neonatal cortisol treatment reportedly decreases levels of growth hormone, growth hormone-releasing factor, and thyroid-stimulating hormone (Sawano et al., 1969). The possibility that thyroid hormone levels may be reduced in cortisoltreated rats is particularly relevant since thyroid hormone is required for normal myelin formation in rat brain (Balazs et al., 1969).

Our observations may be relevant to the clinical use of glucocorticoids as anti-inflammatory agents for the treatment of various conditions, including demyelinating diseases. If the effects of glucocorticoids on gliogenesis and myelination in human are like those in rat, then the beneficial anti-inflammatory effects of these steroids must be weighed carefully against possible deleterious effects on myelination and remyelination.

\section{References}

Aguayo, A. J., G. M. Bray and S. C. Perkins (1979) AxonSchwann cell relationships in neuropathies of mutant mice. Ann. N. Y. Acad. Sci. 317: 512-531.

Balazs, R., B. W. L. Brooksbank, A. N. Davison, J. T. Eyars, and D. A. Wilson (1969) The effect of neonatal thyroidectomy on myelination in the rat brain. Brain Res. 15: 219-232.

Bohn, M. C. (1979) Effect of hydrocortisone on neurogenesis in the neonatal rat brain: A morphological and autoradiographic study. Ph.D. dissertation, University of Connecticut, Storrs, CT.

Bohn, M. C., and J. M. Lauder (1978) The effects of neonatal hydrocortisone on rat cerebellar development. An autoradiographic and light microscopic study. Dev. Neurosci. 1: 250266.

Bohn, M. C., and J. M. Lauder (1980) Cerebellar granule cell genesis in the hydrocortisone-treated rat. Dev. Neurosci. 3: 81-89.

Clos, J., M. Selme-Matrat, A. Rabie, and J. Legrand (1975) Effets du cortisol sur la proliferation et la maturation cellulaires dans le cerveau et le cervelet du Rat: Importance de l'age des animaux au debut du traitement. J. Physiol. (Paris) 70: 207-218.

Cotterrell, M. R., R. Balazs, and A. L. Johnson (1972) Effects of corticosteroids on the biochemical maturation of rat brain: Postnatal cell formation. J. Neurochem. 19: 2151-2167.

Dawson, G., and S. M. Kernes (1979) Mechanism of action of hydrocortisone potentiation of sulfogalactosylceramide synthesis in mouse oligodendroglioma clonal cell lines. J. Biol. Chem. 254: 163-167.

DeVellis, J., and D. Inglish (1973) Age-dependent changes in the regulation of glycerolphosphate dehydrogenase in the rat brain and in glial cell line. Prog. Brain Res. 40: 321-330.

Grasso, R. J., and C. E. Johnson (1977) Dose-response relationship between glucocorticoids and growth inhibition in rat glioma monolayer cultures. Proc. Soc. Exp. Biol. Med. 154: 238-241.

Gumbinas, M., M. Oda, and P. Huttenlocher (1973) The effect of corticosteroids on myelination of the developing rat brain. Biol. Neonate 22: 355-366.

Helwig, J. T., and K. A. Council, eds. (1979) SAS User's Guide, SAS Institute, Ralcigh, NC.

Herndon, R. M., D. L. Price, and L. P. Weiner (1977) Regeneration of oligodendroglia during recovery from demyelinating disease. Science 195: 693-694.

Hirose, G., and N. H. Bass (1973) Maturation of oligodendroglia and myelinogenesis in rat optic nerve: A quantitative histochemical study. J. Comp. Neurol. 152: 201-210.

Howard, E. (1973) Hormonal effects on the growth and DNA content of the developing brain. In Biochemistry of the Developing Brain, W. Himwich, ed., pp. 1-68, Marcel Dekker, New York.

Howard, E., and J. A. Benjamins (1975) DNA, ganglioside and sulfatide in brains of rats given corticosterone in infancy, with an estimate of cell loss during development. Brain Res. 92: $73-87$.

Juulink, B. H. J., A. Schousboe, O. S. Jorgensen, and L. Hertz (1981) Induction by hydrocortisone of glutamine synthetase 
in mouse primary astrocyte cultures. J. Neurochem. 36: 136142.

Ludwin, S. K. (1979) An autoradiographic study of cellular proliferation in remyelination of the central nervous system. Am. J. Pathol 95: 683-696.

Ludwin, S. K. (1980) Chronic demyelination inhibits remyelination in the central nervous system. An analysis of contributing factors. Lab. Invest. 43: 382-387.

Matthew, M. A., and D. Duncan (1971) A quantitative study of morphological changes accompanying the initiation and progress of myelin production in the dorsal funiculus of the rat spinal cord. J. Comp. Neurol. 142: 1-22.

Meyer, J. S., and B. S. McEwen (1982) Evidence for glucocorticoid target cells in rat optic nerve. Physicochemical characterization of cytosol binding sites. J. Neurochem. 39: 435442.

Olton, D. S., C. T. Johnson and E. Howard (1974) Impairment of conditioned active avoidance in adult rats given corticosterone in infancy. Dev. Psychobiol. 8: 55-61.

Privat, A., and C. P. Leblond (1972) The subependymal layer and neighboring region in the brain of the young rat. $J$. Comp. Neurol. 146: 277-302.

Salas, M., and S. Schapiro (1970) Hormonal influences upon the maturation of the rat brain's responsiveness to sensory stimuli. Physiol. Behav. 5: 7-11.

Sawano, S., A. Arimura, A. V. Schally, T. W. Redding, and S. Schapiro (1969) Neonatal corticoid administration: Effects upon adult pituitary growth hormone and hypothalamic growth hormone-releasing hormone activity. Acta Endocrinol. (Copenh.) 61: 57-67.

Schapiro, S. (1968) Some physiological, biochemical and behavioral consequences of neonatal hormone administration: cortisol and thyroxine. Gen. Comp. Endocrinol. 10: 214-228.

Sidman, R. L. (1961) Histogenesis of the mouse retina studied with tritiated thymidine. In The Structure of the Eye, G. K. Smelser, ed., pp. 487-505, Academic Press, New York.
Skoff, R. P., D. L. Price, and A. Stocks (1976a) Electron microscopic autoradiographic studies of gliogenesis in rat optic nerve. I. Cell proliferation. J. Comp. Neurol. 169: 291312.

Skoff, R. P., D. L. Price, and A. Stock (1976b) Electron microscopic autoradiographic studies of gliogenesis in rat optic nerve. II. Time of origin. J. Comp. Neurol. 169: 313-334.

Skoff, R. P., D. Toland, and E. Nast (1980) Pattern of myelination and distribution of neuroglial cells along the developing optic system of the rat and rabbit. J. Comp. Neurol. 191: $237-253$.

Smart, I. (1961) The subependymal layer of the mouse brain and its cell production as shown by radioautography after thymidine- $\mathrm{H}^{3}$ injection. J. Comp. Neurol. 116: 325-347.

Tennekoon, G., S. R. Cohen, D. L. Price, and G. M. McKhann (1977) Myelinogenesis in optic nerve. J. Cell Biol. 72: 604616.

Tennekoon, G. I., Y. Kishimoto, I. Singh, G. Nonaka, and J. -M. Bourre (1980) The differentiation of oligodendrocytes in the rat optic nerve. Dev. Biol. 79: 149-158.

Vaughn, J. E. (1969) An electron microscopic analysis of gliogenesis in rat optic nerves. Z. Zellforsch. Mikrosk. Anat. 94: 293-324.

Vaughn, J. E., and A. Peters (1967) Electron microscopy of the early postnatal development of fibrous astrocytes. Am. J. Anat. 121: 131-152.

Vernadakis, A., and D. M. Woodbury (1963) Effect of cortisol on the electroshock seizure thresholds of developing rats. J. Pharmacol. Exp. Ther. 13.9: 110-113.

Waziri, R., and S. K. Sahu (1980) Induction of $2^{\prime}, 3^{\prime}$-cyclic nucleotide $3^{\prime}$-phosphohydrolase and morphological alterations in C6 glioma cells by dexamethasone, (3-butoxy-4-methoxybenzyl)-2-imidazoline and prostaglandin E1. In Vitro 16: 97-102.

Williams, M. A. (1977) Quantitative Methods in Biology, North-Holland, Amsterdam. 